\title{
Some New Perspectives on Global Domination in Graphs
}

\author{
S. K. Vaidya ${ }^{1}$ and R. M. Pandit ${ }^{2}$ \\ ${ }^{1}$ Saurashtra University, Rajkot, Gujarat 360005, India \\ ${ }^{2}$ A. V. Parekh Technical Institute, Rajkot, Gujarat 360001, India \\ Correspondence should be addressed to S. K. Vaidya; samirkvaidya@yahoo.co.in
}

Received 10 May 2013; Accepted 20 July 2013

Academic Editors: P. Garcia-Vazquez and P. E. Jorgensen

Copyright (C) 2013 S. K. Vaidya and R. M. Pandit. This is an open access article distributed under the Creative Commons Attribution License, which permits unrestricted use, distribution, and reproduction in any medium, provided the original work is properly cited.

A dominating set is called a global dominating set if it is a dominating set of a graph $G$ and its complement $\bar{G}$. Here we explore the possibility to relate the domination number of graph $G$ and the global domination number of the larger graph obtained from $G$ by means of various graph operations. In this paper we consider the following problem: Does the global domination number remain invariant under any graph operations? We present an affirmative answer to this problem and establish several results.

\section{Introduction}

The domination in graphs is one of the concepts in graph theory which has attracted many researchers to work on it. Many variants of dominating sets are available in the existing literature. This paper is focused on global domination in graphs.

We begin with simple, finite, and undirected graph $G=$ $(V, E)$ with $|V|=n$. The set $S \subseteq V$ is called a dominating set if $N[S]=V$. A dominating set $S$ is called a minimal dominating set (MDS) if no proper subset $S^{\prime}$ of $S$ is a dominating set.

The minimum cardinality of a dominating set in $G$ is called the domination number of $G$ denoted by $\gamma(G)$, and the corresponding dominating set is called a $\gamma$-set of $G$.

The complement $\bar{G}$ of $G$ is the graph with vertex set $V$ in which two vertices are adjacent in $\bar{G}$ if and only if they are not neighbors in $G$.

A dominating set $S$ of $G$ is called a global dominating set if it is also a dominating set of $\bar{G}$. The global domination number $\gamma_{g}(G)$ is the minimum cardinality of a global dominating set of $G$. The concept of global domination in a graph was introduced by Sampathkumar [1]. This concept is remained in focus of many researchers. For example, the global domination number of boolean function graph is discussed by Janakiraman et al. [2]. The NP completeness of global domination problems is discussed by Carrington [3] and by Carrington and Brigham [4]. The global domination number for the larger graphs obtained from the given graph is discussed by Vaidya and Pandit [5] while Kulli and Janakiram [6] have introduced the concept of total global dominating sets. The discussion on global domination in graphs of small diameters is carried out by Gangadharappa and Desai [7].

The wheel $W_{n}$ is defined to be the join $C_{n-1}+K_{1}$ where $n \geq$ 4. The vertex corresponding to $K_{1}$ is known as apex vertex, and the vertices corresponding to cycle $C_{n-1}$ are known as rim vertices.

Duplication of an edge $e=u v$ of a graph $G$ produces a new graph $G_{1}$ by adding an edge $e^{\prime}=u^{\prime} v^{\prime}$ such that $N(u)=N\left(u^{\prime}\right)$ and $N(v)=N\left(v^{\prime}\right)$.

The shadow graph $D_{2}(G)$ of a connected graph $G$ is constructed by taking two copies of $G$, say $G^{\prime}$ and $G^{\prime \prime}$. Join each vertex $u^{\prime}$ in $G^{\prime}$ to the neighbors of the corresponding vertex $u^{\prime \prime}$ in $G^{\prime \prime}$.

A vertex switching $G_{v}$ of a graph $G$ is the graph obtained by taking a vertex $v$ of $G$, removing all the edges incident to $v$ and adding edges joining $v$ to every vertex not adjacent to $v$ in $G$.

For the various graph theoretic notations and terminology we follow West [8] while the terms related to the concept of domination are used in the sense of Haynes et al. [9].

Here we consider the problem: Does the global domination number remain invariant under any graph operations? We present here an affirmative answer to this question for the graphs obtained by various graph operations on $C_{n}$ and $W_{n}$. Moreover, we obtain the domination number and the global domination number for the shadow graph of $P_{n}$ and 
the graphs obtained by switching of a vertex in $C_{n}$ as well as in $W_{n}$.

\section{Main Results}

Theorem 1. If $C_{n}^{\prime}$ is a graph obtained by duplication of an edge in $C_{n}(n \neq 3, n \neq 5)$ by an edge then every $\gamma$-set of $C_{n}^{\prime}$ is a global dominating set of $C_{n}^{\prime}$ and $\gamma\left(C_{n}^{\prime}\right)=\gamma_{g}\left(C_{n}^{\prime}\right)=\lceil n / 3\rceil$. Furthermore, the global domination number remains invariant under the operation of duplication of an edge for $C_{n}$.

Proof. Let $C_{n}^{\prime}$ be a graph obtained by duplication of an edge in $C_{n}(n \neq 3, n \neq 5)$. Without loss of generality, let the edge $e=$ $v_{1} v_{2}$ of $C_{n}$ be duplicated by an edge $e^{\prime}=v_{1}^{\prime} v_{2}^{\prime}$.

Let $S$ be a $\gamma$-set of $C_{n}^{\prime}$. Then $S$ is a dominating set of $C_{n}^{\prime}$. Now, any two distinct vertices $v_{i}$ and $v_{j}$ with $d\left(v_{i}, v_{j}\right) \neq 2$ in $C_{n}^{\prime}$ are enough to dominate $\overline{C_{n}^{\prime}}$ because the vertices which are not in $N\left[v_{i}\right]$ must belong to $N\left[v_{j}\right]$ in $\overline{C_{n}^{\prime}}$. Since every $\gamma$-set of $C_{n}^{\prime}$ contains at least two such vertices $v_{i}$ and $v_{j}$, it is a dominating set of $\overline{C_{n}^{\prime}}$. Hence, every $\gamma$-set of $C_{n}^{\prime}$ is a dominating set of $C_{n}^{\prime}$ as well as of $\overline{C_{n}^{\prime}}$. Consequently, every $\gamma$-set of $C_{n}^{\prime}$ is a global dominating set of $C_{n}^{\prime}$.

Now, consider a $\gamma$-set of $C_{n}^{\prime}$ :

$$
S=\left\{\begin{array}{lr}
\left\{v_{3}, v_{6}, v_{9}, \ldots, v_{3 j}\right\} & \text { if } n \equiv 0(\bmod 3), \\
\left\{v_{3}, v_{6}, v_{9}, \ldots, v_{3 j}\right\} \cup\left\{v_{n}\right\} & \text { if } n \equiv 1 \text { or } 2(\bmod 3) \\
& \text { for } 1 \leq j \leq\left\lfloor\frac{n}{3}\right\rfloor .
\end{array}\right.
$$

Since $d\left(v_{3}\right)=3=d\left(v_{n}\right)=\Delta\left(C_{n}^{\prime}\right), S$ must contain $v_{3}$ and $v_{n}$ for minimum cardinality. Also, to retain the minimum cardinality of $S$, it must contain the vertices $v_{3 j}(2 \leq j \leq$ $\lfloor n / 3\rfloor)$. That is, $|S| \geq\lceil n / 3\rceil$. Now, $S$ being a $\gamma$-set of $C_{n}^{\prime}$ is a global dominating set of $C_{n}^{\prime}$ with minimum cardinality $\lceil n / 3\rceil$. This implies that $\gamma\left(C_{n}^{\prime}\right)=\gamma_{g}\left(C_{n}^{\prime}\right)=\lceil n / 3\rceil$. Also, as reported in Sampathkumar [1], $\gamma_{g}\left(C_{n}\right)=\lceil n / 3\rceil(n \neq 3, n \neq 5)$. Hence, $\gamma_{g}\left(C_{n}\right)=\gamma_{g}\left(C_{n}^{\prime}\right)=\lceil n / 3\rceil(n \neq 3, n \neq 5)$. Thus, the global domination number remains invariant under the operation of duplication of an edge by an edge in $C_{n}$.

Theorem 2. If $G$ is the graph obtained by duplicating each edge of $C_{n}$ by an edge then every $\gamma$-set of $G$ is a global dominating set of $G$ and $\gamma(G)=\gamma_{g}(G)=n$.

Proof. Let $S$ be a $\gamma$-set of $G$. Then $S$ is a dominating set of $G$. Now, any two adjacent vertices $v_{i}$ and $v_{j}$ in $G$ are enough to dominate $\bar{G}$ because the vertices which are not in $N\left[v_{i}\right]$ in $\bar{G}$ must belong to $N\left[v_{j}\right]$ in $\bar{G}$. Since every $\gamma$-set of $G$ contains at least two such vertices $v_{i}$ and $v_{j}$, every $\gamma$-set of $G$ is a dominating set of $\bar{G}$. Hence, every $\gamma$-set of $G$ is a dominating set of $G$ as well as of $\bar{G}$. This implies that every $\gamma$-set of $G$ is a global dominating set of $G$.

Let $v_{1}, v_{2}, \ldots, v_{n}$ be the vertices of $C_{n}$. Consider a $\gamma$-set of $G, S=\left\{v_{1}, v_{2}, \ldots, v_{n}\right\}$. $S$ being a $\gamma$-set of $G$ is a dominating set of $G$ with minimum cardinality. Moreover, since $v_{1}, v_{2}, \ldots, v_{n}$ are the vertices of maximum degree in $G$ and from the nature of the graph $G$, it is clear that $S=\left\{v_{1}, v_{2}, \ldots, v_{n}\right\}$ is of minimum cardinality. Hence, $S$ is a $\gamma$-set of $G$ with minimum cardinality $n$. Therefore, $\gamma(G)=n$. Now, $S$ being a $\gamma$-set of $G$ is a global dominating set of $G$ with minimum cardinality $n$ which implies that $\gamma(G)=\gamma_{g}(G)=n$ as required.

The following Theorem 3 can be proved by the arguments analogous to the above Theorem 2 .

Theorem 3. If $G$ is the graph obtained by duplicating each edge of $P_{n}$ by an edge then every $\gamma$-set of $G$ is a global dominating set of $G$ and $\gamma(G)=\gamma_{g}(G)=n$.

Theorem 4. If $W_{n}^{\prime}$ is a graph obtained by duplicating an edge of $W_{n}$ by an edge then

$$
\gamma_{g}\left(W_{n}\right)=\gamma_{g}\left(W_{n}^{\prime}\right)= \begin{cases}4 & \text { if } n=4 \\ 3 & \text { otherwise }\end{cases}
$$

That is, the global domination number remains invariant under the operation of duplication of an edge in $W_{n}$.

Proof. Let $V\left(W_{n}\right)=\left\{v_{1}, v_{2}, \ldots, v_{n-1}\right\} \cup\{c\}$, where $c$ is the apex vertex of $W_{n}$.

It is easy to observe that

$$
\gamma_{g}\left(W_{n}\right)= \begin{cases}4 & \text { if } n=4 \\ 3 & \text { otherwise. }\end{cases}
$$

Case I (When a rim edge of $W_{n}$ is duplicated by an edge). Without loss of generality, let the rim edge $e=v_{1} v_{2}$ of $W_{n}$ be duplicated by an edge $e^{\prime}=v_{1}^{\prime} v_{2}^{\prime}$.

For $n=4$, since the vertex $c$ is adjacent to each vertex of $W_{n}^{\prime}$, it must belong to any global dominating set of $W_{n}^{\prime}$. Moreover, any two vertices are adjacent to third vertex other than $c$ in $W_{n}^{\prime}$. Therefore, any global dominating set of $W_{n}^{\prime}$ must contain at least four vertices including $c$ which implies that $\gamma_{g}\left(W_{n}^{\prime}\right)=4$.

For $n>4$, the vertex $c$ dominates $W_{n}^{\prime}$ while the vertex $c$ and any two adjacent rim vertices of $W_{n}$ are enough to dominate $\overline{W_{n}^{\prime}}$. Therefore, any global dominating set of $W_{n}^{\prime}$ must contain at least three vertices of $W_{n}^{\prime}$. This shows that $\gamma_{g}\left(W_{n}^{\prime}\right)=3$.

Thus,

$$
\gamma_{g}\left(W_{n}^{\prime}\right)= \begin{cases}4 & \text { if } n=4 \\ 3 & \text { otherwise. }\end{cases}
$$

Case II (When a spoke edge of $W_{n}$ is duplicated by an edge). Without loss of generality, let the spoke edge $e=c v_{1}$ be duplicated by an edge $e^{\prime}=c^{\prime} v_{1}^{\prime}$.

For $n=4$, any two vertices in $W_{n}^{\prime}$ are adjacent to the third vertex and also any three vertices are adjacent to the fourth vertex in $W_{n}^{\prime}$. Therefore, any global dominating set of $W_{n}^{\prime}$ must contain at least four vertices which implies that $\gamma_{g}\left(W_{n}^{\prime}\right)=4$.

For $n>4$, clearly any global dominating set of $W_{n}^{\prime}$ must contain either $c$ or $c^{\prime}$ to achieve its minimum cardinality. 
Moreover, any two adjacent rim vertices of $W_{n}$ and the vertex $c$ are enough to dominate $\overline{W_{n}^{\prime}}$ and they also dominate $W_{n}^{\prime}$. This implies that $\gamma_{g}\left(W_{n}^{\prime}\right)=3$.

Thus,

$$
\gamma_{g}\left(W_{n}^{\prime}\right)= \begin{cases}4 & \text { if } n=4 \\ 3 & \text { otherwise }\end{cases}
$$

Hence, we have proved that

$$
\gamma_{g}\left(W_{n}\right)=\gamma_{g}\left(W_{n}^{\prime}\right)= \begin{cases}4 & \text { if } n=4 \\ 3 & \text { otherwise }\end{cases}
$$

That is, the global domination number remains invariant under the operation of duplication of an edge in $W_{n}$.

Theorem 5. Every $\gamma$-set of $D_{2}\left(P_{n}\right)$ is a global dominating set of $D_{2}\left(P_{n}\right)$, and

$$
\begin{aligned}
\gamma\left(D_{2}\left(P_{n}\right)\right) & =\gamma_{g}\left(D_{2}\left(P_{n}\right)\right) \\
& = \begin{cases}\frac{n}{2} & \text { if } n \equiv 0(\bmod 4) \\
\left\lfloor\frac{n}{2}\right\rfloor+1 & \text { otherwise. }\end{cases}
\end{aligned}
$$

Proof. Consider two copies of $P_{n}$. Let $v_{1}, v_{2}, \ldots, v_{n}$ be the vertices of the first copy of $P_{n}$ and $u_{1}, u_{2}, \ldots, u_{n}$ the vertices of the second copy of $P_{n}$.

If $S$ is a $\gamma$-set of $D_{2}\left(P_{n}\right)$ then $S$ is a dominating set of $D_{2}\left(P_{n}\right)$. Now, any two adjacent vertices $v_{i}$ and $v_{j}$ of $D_{2}\left(P_{n}\right)$ are enough to dominate $\overline{D_{2}\left(P_{n}\right)}$ because the vertices which are not in $N\left[v_{i}\right]$ in $\overline{D_{2}\left(P_{n}\right)}$ must belong to $N\left[v_{j}\right]$ in $\overline{D_{2}\left(P_{n}\right)}$. Since every $\gamma$-set of $G$ contains at least two such vertices $v_{i}$ and $v_{j}$, every $\gamma$-set of $D_{2}\left(P_{n}\right)$ is a dominating set of $\overline{D_{2}\left(P_{n}\right)}$. Hence, every $\gamma$-set of $D_{2}\left(P_{n}\right)$ is a dominating set of $D_{2}\left(P_{n}\right)$ as well as of $\overline{D_{2}\left(P_{n}\right)}$. This implies that every $\gamma$-set of $D_{2}\left(P_{n}\right)$ is a global dominating set of $D_{2}\left(P_{n}\right)$.

Case $I(n=2,3)$. For $D_{2}\left(P_{2}\right)$ and $D_{2}\left(P_{3}\right)$, clearly $S=$ $\left\{v_{1}, v_{2}\right\}$ and $S=\left\{v_{2}, v_{3}\right\}$ are $\gamma$-sets as well as global dominating sets with minimum cardinality respectively. Therefore, $\gamma\left(D_{2}\left(P_{n}\right)\right)=\gamma_{g}\left(D_{2}\left(P_{n}\right)\right)=2=\lfloor n / 2\rfloor+1$ for $n=2,3$.

Case II $(n \geq 4)$. (i) For $n \equiv 0(\bmod 4)$ (i.e., $n=4 k, k \in N)$, consider a $\gamma$-set $S=\left\{v_{2+4 i}, v_{3+4 i} / 0 \leq i \leq k-1\right\}$ where $|S|=$ $n / 2$.

(ii) For $n \equiv 1(\bmod 4)$ (i.e., $n=4 k+1, k \in N)$, consider a $\gamma$-set $S=\left\{v_{2+4 i}, v_{3+4 i} / 0 \leq i \leq k-1\right\} \cup\left\{v_{n-1}\right\}$ where $|S|=$ $\lfloor n / 2\rfloor+1$.

(iii) For $n \equiv 2$ or $3(\bmod 4)$ (i.e., $n=4 k+2$ or $n=4 k+$ $3, k \in N)$, consider a $\gamma$-set $S=\left\{v_{2+4 i}, v_{3+4 i} / 0 \leq i \leq k-1\right\} \cup$ $\left\{v_{n-2}, v_{n-1}\right\}$ where $|S|=\lfloor n / 2\rfloor+1$.

Now, $S$ being a $\gamma$-set of $D_{2}\left(P_{n}\right)$ is a global dominating set of $D_{2}\left(P_{n}\right)$ with minimum cardinality implying that $|S|=$ $\gamma\left(D_{2}\left(P_{n}\right)\right)=\gamma_{g}\left(D_{2}\left(P_{n}\right)\right)$.
Thus, we have proved that

$$
\gamma\left(D_{2}\left(P_{n}\right)\right)=\gamma_{g}\left(D_{2}\left(P_{n}\right)\right)= \begin{cases}\frac{n}{2} & \text { if } n \equiv 0(\bmod 4) \\ \left\lfloor\frac{n}{2}\right\rfloor+1 & \text { otherwise. }\end{cases}
$$

Theorem 6. If $G_{v}$ is a graph obtained by switching of a vertex in cycle $C_{n}(n \geq 8)$ then $\gamma\left(G_{v}\right)=\gamma_{g}\left(G_{v}\right)=3$.

Proof. Let $v_{1}, v_{2}, \ldots, v_{n}$ be the successive vertices of $G=C_{n}$ and $G_{v}$ denotes the graph obtained by switching of a vertex $v$ of $G$. Without loss of generality, let the switched vertex be $v_{1}$.

Consider a set $S=\left\{v_{1}, v_{2}, v_{n}\right\}$. Then $S$ is a dominating set of $G_{v}$ as all the vertices except the pendant vertices, namely, $v_{2}$ and $v_{n}$, are in $N\left[v_{1}\right]$ while $v_{2}$ and $v_{n}$ are already in $S$. Moreover, the set $S$ is a minimal dominating set of $G_{v}$ because for any $v_{i} \in S$, the set $S-\left\{v_{i}\right\}$ does not dominate the vertex $v_{i}$ of $G_{v}$. Furthermore, the vertex $v_{1}$ dominates $(n-2)$ vertices of $G_{v}$ and the remaining two vertices are pendant vertices. Therefore, at least three vertices are required to dominate $G_{v}$, and hence $|S| \geq 3$. Thus, $S$ being a minimal dominating set with minimum cardinality is a $\gamma$-set of $G_{v}$ which implies that $\gamma\left(G_{v}\right)=3$

Now, we claim that the pendant vertices in $G_{v}$ are enough to dominate remaining vertices of $\overline{G_{v}}$. Since the vertex which is not in $N\left[v_{2}\right]$ in $\overline{G_{v}}$ must belong to $N\left[v_{n}\right]$ in $\overline{G_{v}}$, any $S \subseteq V$ containing $v_{2}$ and $v_{n}$ will be a dominating set of $\overline{G_{v}}$. Thus, $S$ is a dominating set of $G_{v}$ as well as of $\overline{G_{v}}$. This implies that $S$ is a global dominating set of $G_{v}$.

Since $S$ is a $\gamma$-set of $G_{v}$ as above, it is of minimum cardinality. Therefore, $\gamma_{g}\left(G_{v}\right)=3$. Thus, $\gamma\left(G_{v}\right)=\gamma_{g}\left(G_{v}\right)=3$ as required.

Theorem 7. If $G_{v}$ is a graph obtained by switching of a rim vertex in a wheel $W_{n}$ then

$$
\gamma_{g}\left(G_{v}\right)= \begin{cases}2 & \text { if } n=4,5 \\ 3 & \text { otherwise. }\end{cases}
$$

Proof. Let $v_{1}, v_{2}, \ldots, v_{n-1}$ be the successive rim vertices of $G=W_{n}$ and $G_{v}$ denotes the graph obtained by switching of the vertex $v$ of $G$. Without loss of generality, let the switched vertex be $v_{1}$. Let $c$ be the apex vertex of $W_{n}$.

Case $I(n=4,5)$. It is easy to observe that $S=\left\{v_{1}, v_{3}\right\}$ is a global dominating set of $G_{v}$ with minimum cardinality. Therefore, $\gamma_{g}\left(G_{v}\right)=2$ for $n=4,5$.

Case II $(n \geq 6)$. Consider a set $S=\left\{c, v_{3}, v_{4}\right\}$. Then, $S$ is a dominating set of $G_{v}$ as all the vertices except the vertex $v_{1}$ are in $N[c]$ in $G_{v}$ while $v_{1}$ is dominated by $v_{3}$. Now, the vertex $v_{3}$ dominates all the vertices of $\overline{G_{v}}$ except the vertices $v_{1}, v_{2}, v_{4}$, and $c$. But the vertices $v_{1}$ and $v_{2}$ are dominated by the vertices $c$ and $v_{4}$ in $\overline{G_{v}}$ respectively while the vertices $v_{4}$ and $c$ are already in $S$. Therefore, $S$ is a dominating set of $\overline{G_{v}}$. Hence, $S$ is a dominating set of $G_{v}$ as well as of $\overline{G_{v}}$. That is, 
$S$ is a global dominating set of $G_{v}$. Moreover, two vertices, namely, $c$ and either of the vertices from $v_{3}$ and $v_{4}$ are enough to dominate $G_{v}$. Since any two vertices in $G_{v}$ are adjacent to the third vertex in $G_{v}$, at least three vertices are essential to dominate $\overline{G_{v}}$. This implies that $\gamma_{g}\left(G_{v}\right)=3$. Thus, we have proved that

$$
\gamma_{g}\left(G_{v}\right)= \begin{cases}2 & \text { if } n=4,5 \\ 3 & \text { otherwise }\end{cases}
$$

Theorem 8. If $G_{v}$ is the graph obtained by switching of the apex vertex in wheel $W_{n}$ then $\gamma\left(G_{v}\right)=\gamma_{g}\left(G_{v}\right)=\lceil(n-1) / 3\rceil+1$.

Proof. Let $v_{1}, v_{2}, \ldots, v_{n-1}$ be the successive rim vertices of $G=$ $W_{n}$ and let $G_{v}$ denote the graph obtained by switching of the vertex $v$ of $G$. Let the switched vertex be the apex vertex $c$ of $W_{n}$.

Because $c$ is adjacent to every other vertex in $G, c$ is not adjacent to any other vertex in $G_{v}$. Therefore, any dominating set for $G_{v}$ must contain $c$. This implies that any global dominating set of $G_{v}$ must contain $c$.

Case $I(n=4,6)$. It is easy to observe that $S=\left\{c, v_{1}\right\}$ and $S=\left\{c, v_{1}, v_{3}\right\}$ are $\gamma$-sets as well as global dominating sets of $G_{v}$ of $W_{4}$ and $W_{6}$ respectively with minimum cardinality.

Therefore,

$$
\gamma\left(G_{v}\right)=\gamma_{g}\left(G_{v}\right)= \begin{cases}2=\left\lceil\frac{(4-1)}{3}\right\rceil+1 & \text { for } n=4, \\ 3=\left\lceil\frac{(6-1)}{3}\right\rceil+1 & \text { for } n=6 .\end{cases}
$$

Thus, $\gamma\left(G_{v}\right)=\gamma_{g}\left(G_{v}\right)=\lceil(n-1) / 3\rceil+1$ for $n=4,6$.

Case II $(n \neq 4,6)$. The vertex $c$ will be the isolated vertex in $G_{v}$. Hence, $G_{v}=C_{n-1} \cup K_{1}$. Therefore, $\gamma\left(G_{v}\right)=\gamma\left(C_{n-1}\right)+1$. But as reported in Sampathkumar [1], $\gamma\left(C_{n-1}\right)=\lceil(n-1) / 3\rceil$. This implies that $\gamma\left(G_{v}\right)=\lceil(n-1) / 3\rceil+1$.

Because every dominating set of $G_{v}$ must contain $c$ and $c$ is enough to dominate $\overline{G_{v}}, \gamma\left(G_{v}\right)=\gamma_{g}\left(G_{v}\right)$.

Hence, $\gamma\left(G_{v}\right)=\gamma_{g}\left(G_{v}\right)=\lceil(n-1) / 3\rceil+1$ for $n \neq 4,6$.

Thus, we have proved that $\gamma\left(G_{v}\right)=\gamma_{g}\left(G_{v}\right)=\lceil(n-1) / 3\rceil+$ 1 as required.

\section{Concluding Remarks}

The concept of global domination is remarkable as it relates the dominating sets of a graph and its complement. We have explored this concept in the context of some graph operations and also have investigated the domination number and the global domination number for the larger graph obtained by some graph operations on a given graph. The invariance parameter for global domination is also explored.

\section{Acknowledgment}

The authors are highly thankful to the anonymous referees for their kind comments and fruitful suggestions on the first draft of this paper.

\section{References}

[1] E. Sampathkumar, "The global domination number of a graph," Journal of Mathematical and Physical Sciences, vol. 23, no. 5, pp. 377-385, 1989.

[2] T. N. Janakiraman, S. Muthammai, and M. Bhanumathi, "Global domination and neighborhood numbers in Boolean function graph of a graph," Mathematica Bohemica, vol. 130, no. 3, pp. 231-246, 2005.

[3] J. R. Carrington, Global domination of factors of a graph [Ph.D. dissertation], University of Central Florida, 1992.

[4] J. R. Carrington and R. C. Brigham, "Global domination of simple factors," Congressus Numerantium, vol. 88, pp. 161-167.

[5] S. K. Vaidya and R. M. Pandit, "Some new results on global dominating sets," ISRN Discrete Mathematics, vol. 2012, Article ID 852129, 6 pages, 2012.

[6] V. R. Kulli and B. Janakiram, "The total global domination number of a graph," Indian Journal of Pure and Applied Mathematics, vol. 27, no. 6, pp. 537-542, 1996.

[7] D. B. Gangadharappa and A. R. Desai, "On the dominating of a graph and its complement," Journal of Mathematics and Computer Science, vol. 2, no. 2, pp. 222-233, 2011.

[8] D. B. West, Introduction to Graph Theory, Prentice Hall, New Delhi, India, 1996.

[9] T. W. Haynes, S. T. Hedetniemi, and P. J. Slater, Fundamentals of Domination in Graphs, vol. 208 of Monographs and Textbooks in Pure and Applied Mathematics, Marcel Dekker, New York, NY, USA, 1998. 


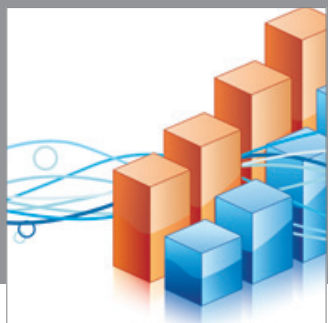

Advances in

Operations Research

mansans

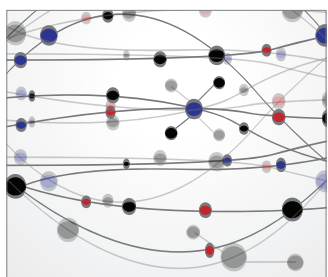

The Scientific World Journal
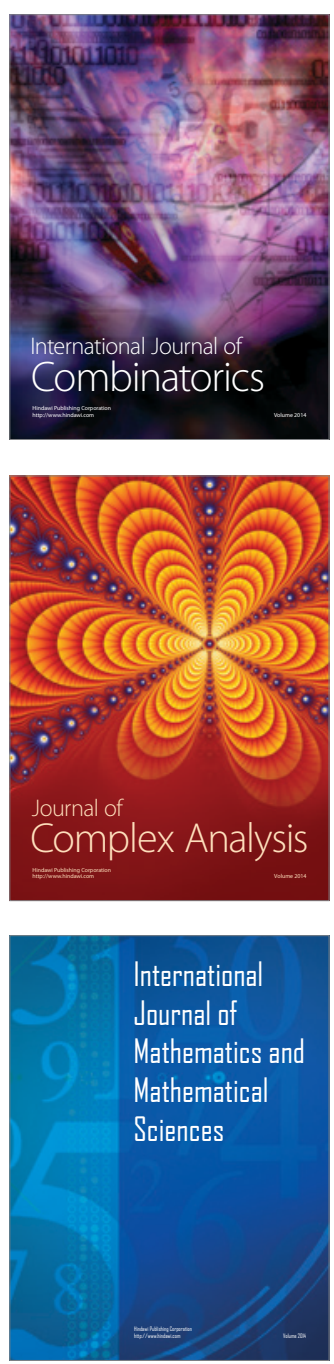
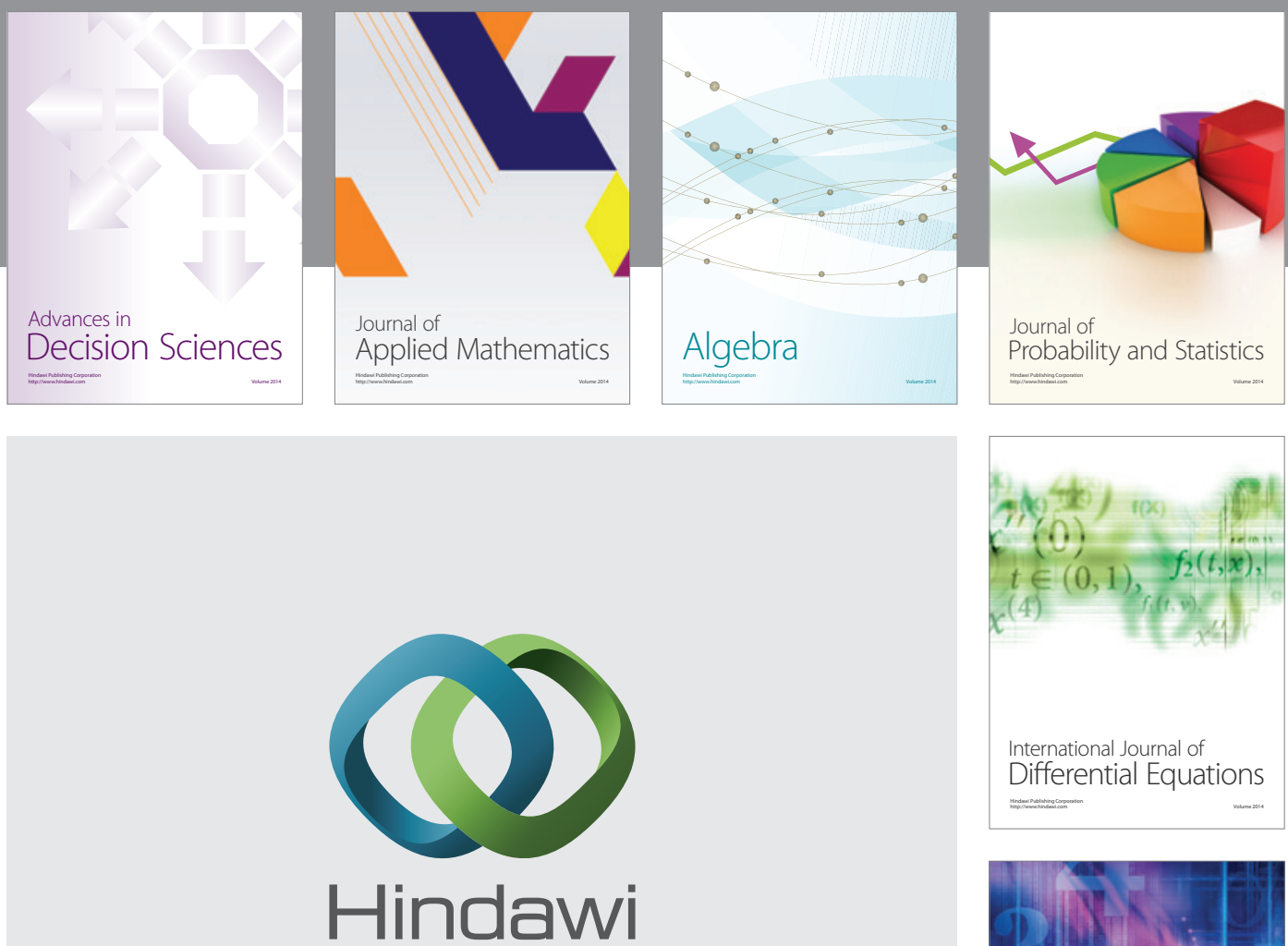

Submit your manuscripts at http://www.hindawi.com
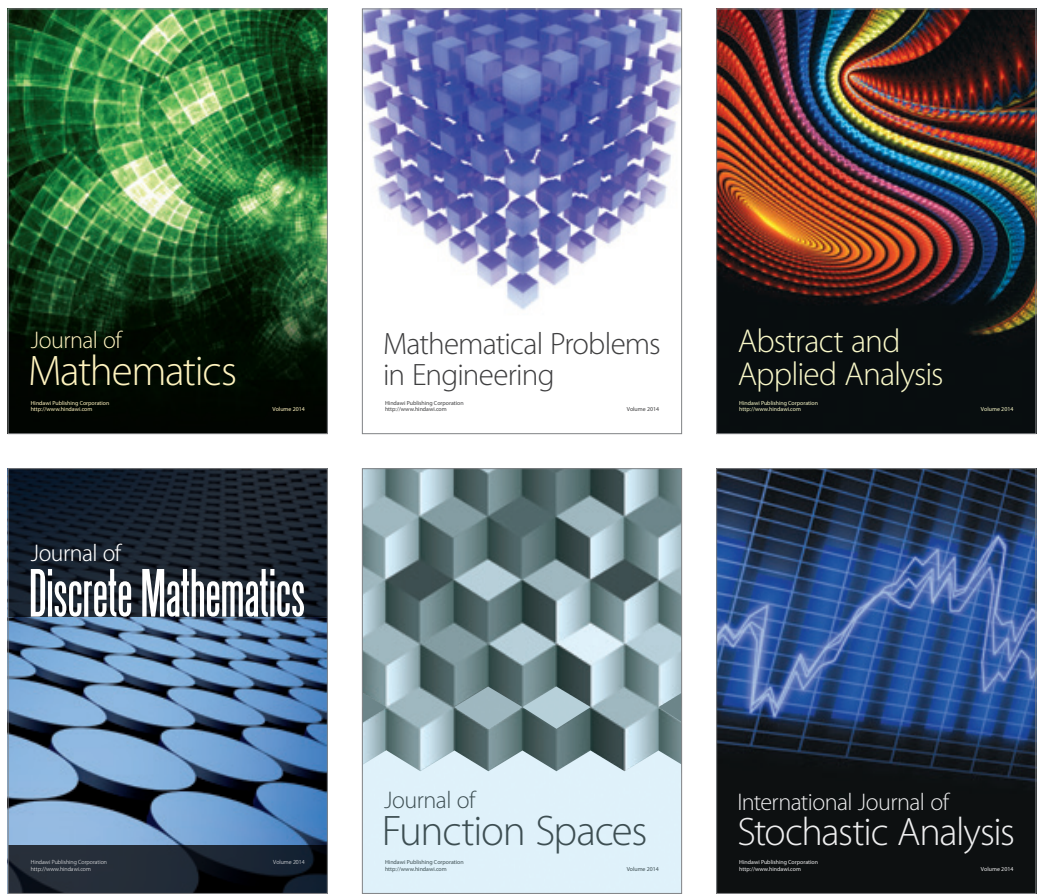

Journal of

Function Spaces

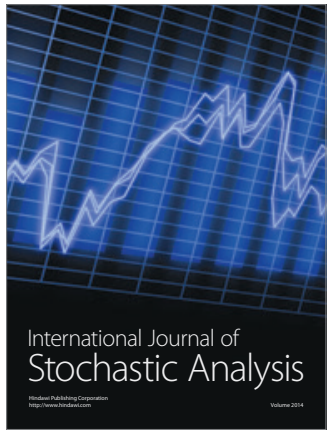

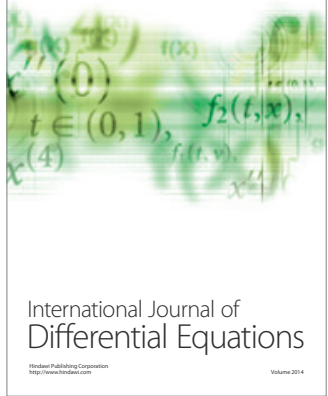
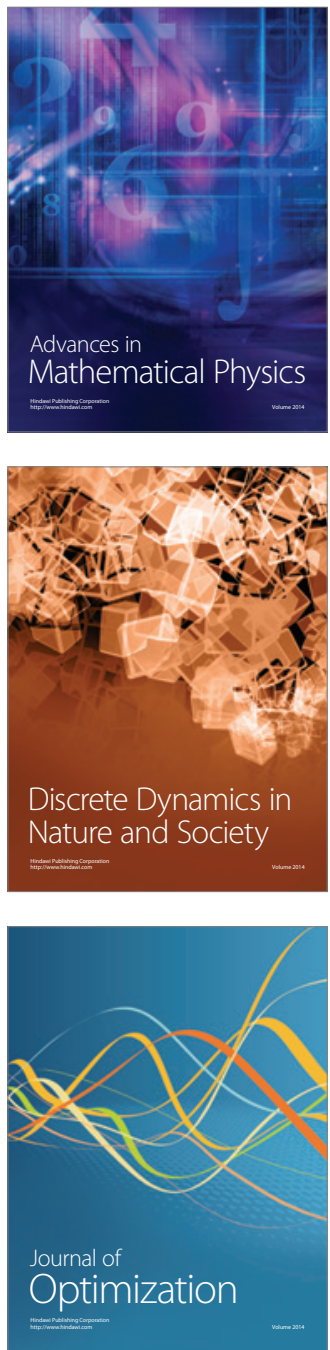